DOI: $10.2478 / \mathrm{v} 10014-009-0016-y$

Agrovoc descriptors: Thysanoptera; encarsia formosa; identification; classification; geographical distribution; trialeurodes vaporariorum; biological control; pest control; natural enemies; indigenous organisms; greenhouses; plant protection

Agris category code: $\mathrm{H} 10$

\title{
Distribution of Thysanoptera species and their host plants in Croatia
}

\author{
Emilija RASPUDIĆ ${ }^{1}$, Marija IVEZIĆ ${ }^{2}$, Mirjana BRMEŽ ${ }^{3}$, Stanislav TRDAN ${ }^{4}$
}

Received July 27, 2009; accepted August 24, 2009.

Delo je prispelo 27. julija 2009, sprejeto 24. avgusta 2009.

\begin{abstract}
Sampling of thrips species and their host plants were carried out from 1993 to 1996 on 111 localities in Croatia. Samples of thrips were taken from 235 different plant species. 33 thrips species from suborder Terebrantia and 14 thrips species from suborder Tubulifera were found in research. The most abundant species were onion thrips (Thrips tabaci) and flower thrips (Frankliniella intonsa), which were found on host plants from 30 and 29 botanical families, respectively. Six new species for Croatia was recorded: Aeolothrips melaleucus, Oxythrips bicolor, Stenothrips graminum, Thrips linarius, Liothrips vaneeckei, and Poecilothrips albopictus.
\end{abstract}

Key words: thrips, Thysanoptera, host plants, sampling, Croatia

\section{RESARJI (Thysanoptera) IN NJIHOVE GOSTITELJSKE RASTLINE NA HRVAŠKEM \\ IZVLEČEK}

V obdobju 1993-1996 smo na 111 lokacijah na Hrvaškem vzročili resarje (Thysanoptera) na različnih vrstah gostiteljskih rastlin. Vzorce resarjev smo nabrali na 235 vrstah rastlin. 33 vrst resarjev, najdenih $\mathrm{v}$ raziskavi, je pripadalo podredu Terebrantia, 14 vrst pa podredu Tubulifera. Najbolj razširjeni vrsti sta bili tobakov resar (Thrips tabaci), ki smo ga našli na rastlinskih vrstah iz 30 botaničnih družin, in resar Frankliniella intonsa, ki je bil ugotovljen na gostiteljskih rastlinah iz 29 botaničnih družin. V raziskavi smo potrdili razširjenost 6 vrst resarjev, ki na Hrvaškem dotlej še niso bile najdene: Aeolothrips melaleucus, Oxythrips bicolor, Stenothrips graminum, Thrips linarius, Liothrips vaneeckei in Poecilothrips albopictus.

Ključne besede: resarji, Thysanoptera, gostiteljske rastline, vzorčrenje, Hrvaška

\section{INTRODUCTION}

The order Thysanoptera is homogenous group of insects with characteristic wings - they have long fringe and very poor nervature. The adults are only few $\mathrm{mm}$ long and their detection is not easy. Up to date, more than 5000 species from two suborders - Terebrantia and Tubulifera - and 8 families - Merothripidae, Aeolothripidae, Heterothripidae, Adiheterothripidae,
Thripidae, Uzelothripidae, Fauriellidae, and Phlaeothripidae, are described. $93 \%$ of species belong to the families Thripidae and Phlaeothripidae (Mound, 1997) and their representatives are also the most common in Croatia and its neighbouring countries (Trdan et al., 2003). The most important pests from Thysanoptera order - e.g. Frankliniella occidentalis

\footnotetext{
1 Josip Juraj Strossmayer University in Osijek, Faculty of Agriculture, Trg Svetog Trojstva 3, HR-31000 Osijek, Croatia; email: emilija.raspudic@pfos.hr

2 ibid.

3 ibid.

${ }^{4}$ University of Ljubljana, Biotechnical Faculty, Dept. of Agronomy, Jamnikarjeva 101, SI-1111 Ljubljana
} 
(Pergande), Thrips tabaci Lindeman, Thrips palmi Karny and Scirtothrips dorsalis Hood - belong to Thripidae family (Mound, 1997). In Croatia, up to now thrips fauna was investigated by Kovačević (1964), zur Strassen (1981, 1984), Ciglar et al. (1984, 1990), Šimala (1991), Raspudić (1996) and Andjus (1997).

Knowledge on host plants of thrips is important, since many polyphagous species and viruses they transmit can survive on different wild-growing plants also out of growing season of the plants (in most cases the cultivated plants), in which this pests caused the highest damage (Jenser et al., 2007). Until now, in Croatia and in some of its neighbouring countries many authors investigated distribution of Thysanoptera and their host plants: Jenser (1986, 1990), Jenser and Tusnádi (1989), Jenser and Balogh (1992), Masten (1983), Ciglar et al. (1984, 1990), Janežič (1991), Šimala (1991), Janežič and Maček (1993), Raspudić (1999), and Trdan (2002, 2003). The aim of this research was to identify thrips species and their host plants in Croatia, since the previous data from the same country was connected only with the individual thrips species.

\section{MATERIALS AND METHODS}

Monitoring of thrips fauna was carried out in Croatia from 1993 to 1996. Total of 662 samples - most of them were collected outdoors - from 235 different plant species were taken from 111 localities. Thrips sampling was done using standard thysanopterological instruments (Raspudić, 1996; Trdan, 2002). The samples were taken from agricultural crops, plants in the garden, channels, meadows, bushes and forests. Determination of thrips was carried out in the Entomological
Laboratory (Josip Juraj Strossmayer University in Osijek, Faculty of Agriculture) on the base of 1058 permanent slides. Determination was made according to morphological keys of Jenser (1982), Okumura and Papp (1991), and Moritz (1994). Determination of plant species was done by Prof. Ana Skender (Josip Juraj Strossmayer University in Osijek, Faculty of Agriculture), according to morphological keys of Ehrendorfer (1973) and Domac (1994).

\section{RESULTS}

Results on determination of thrips species and their hosts plants are presented in chapters 3.1 and 3.2, in which Thysanoptera and their host plant species are presented at family levels.

\subsection{Suborder Terebrantia}

\subsubsection{Family Aeolothripidae}

3.1.1.1 Aeolothrips intermedius Bagnall, 1934

Apiaceae: Daucus carota L.

Asteraceae: Cirsium arvensis (L.) Scop., Helianthus annuus L., Centaurea cristata Bartl.

Brassicaceae: Sinapis arvensis L., Rorippa austriaca (Cr.) Bess., Raphanus sativus L.

Caprifoliaceae: Sambucus nigra L.

Chenopodiaceae: Beta vulgaris L. var. saccharifera Lange,

Fabaceae: Trifolium campestre Schreb, Glycine max (L.) Merr., Trifolium pratense L., Phaseolus vulgarea L., Trifolium repens L.

Iridaceae: $\quad$ Gladiolus gandavensis van Houtte.

Papaveraceae: Papaver rhoeas L., Papaver somniferum L.

Poaceae: $\quad$ Zea mays L.

Polygonaceae: Rumex obtusifolius L.

Punicaceae: Punica granatum L.

Rosaceae: $\quad$ Rosa canina L.

Solanaceae: Nicotiana tabacum L.
3.1.1.2 Aeolothrips melaleucus Haliday, 1852

Caprifoliaceae: Sambucus ebulus L.

Corylaceae: Corylus avellana L.

Fabaceae: $\quad$ Glycine max (L.) Merr.

Puncaceae: $\quad$ Punica granatum L.

Rosaceae: $\quad$ Prunus persica (L.) Batsch

Vitaceae: Vitis vinifera L.

3.1.1.3 Aeolothrips ericae Bagnall, 1920

Poaceae: $\quad$ Zea mays L.

3.1.1.4 Melanthrips pallidior Priesner, 1919

Chenopodiaceae: Spinacia oleracea L.

Brassicaceae: Raphanus sativus L., Rorippa austriaca (Cr.) Bess.

3.1.1.5 Aeolothripidae larvae

Apiaceae: Daucus carota L.

Asteraceae: Cirsium arvense (L.) Scop., Tripleurospermum inodorum (L.) C.H. Schultz

Brassicaceae: Raphanus landra Moretti

Fabaceae: $\quad$ Coronilla varia L., Medicago sativa L., Trifolium repens L., Dorycnium herbaceum Vill., Melilotus alba Med.

Poaceae: $\quad$ Avena sativa L., Zea mays L., Sorgum bicolor (L.) Moench.

Polygonaceae: Rumex crispus L., Polygonum lapathifolium L.

Rubiaceae: Galium mollugo L. 


\subsubsection{Family Thripidae}

3.1.2.1 Aptinothrips rufus (Haliday, 1836)

Fabaceae: Coronilla varia L.

Poaceae: Bromus sp., Melica transsilvanica Schur, Triticum aestivum L., Lolium perenne L.

3.1.2.2 Aptinothrips stylifer Trybom, 1894

Brassicaceae: Capsella bursa-pastoris (L.) Med.

Fabaceae: Medicago sativa L.

Malvaceae: Malva sylvestris L.

Poaceae: $\quad$ Triticum aestivum L.

Solanaceae: Petunia hybrida

3.1.2.3 Anaphothrips obscurus (O.F. Müller, 1776)

Ranunculaceae: Clematis vitalba L.

3.1.2.4 Ceratothrips ericae (Haliday, 1836)

Apiaceae: Anethum graveolens L., Daucus carota L.

Asteraceae: $\quad$ Inula britannica L., Inula crithmoides L., Calendula officinalis L.,

Cichoriaceae: Crepis setosa Hall.f. Taraxacum officinale Web., Crepis biennis L.,Cichorium intbus L., Sonchus arvensis L., Lactuca sativa L., Picris sp., Picris hieracioides L., Chondrilla juncae L., Sonchum arvensis L., Sonchus sp.

Convolvulaceae: Convolvulus arvensis L.

Cucurbitaceae: Cucurbita pepo L.

Dipsaceae: Scabiosa ochroleuca L.

Iridaceae: Gladiolus gandavensis van Houtte

Lamiaceae: $\quad$ Nepeta cataria L.

Poaceae: $\quad$ Zea mays L., Deschampisia flexuosa (L.) Trin.

Rosaceae: $\quad$ Spirea salicifolia L.

Verbanaceae: Vitex agnus castus L.

3.1.2.5 Chirothrips aculeatus Bagnall, 1927

Fabaceae: $\quad$ Trifolium repens L.

Poaceae: Lolium perenne L.

3.1.2.6 Chirothrips manicatus Haliday, 1836

Asteraceae: $\quad$ Erigeron annuus L.

Brassicaceae: Sinapis arvensis L..

Lamiaceae: Nepeta pannonica L.

Malvaceae: $\quad$ Malva alcea L.

Poaceae: Dactylis glomerata L., Calamagrostis epigeios (L.) Roth, Zea mays L, Lolium perenne L., Sorgum halepense (L.) Pers, Sorgum bicolor (L.) Moench, Festuca heterophylla Lam., Deschampisia flexuosa (L.) Trin.

Rosaceae: $\quad$ Malus sp.
3.1.2.7 Drepanothrips reuteri Uzel, 1895

Asteraceae: Artemisia vulgaris L.

3.1.2.8 Frankliniella intonsa (Trybom, 1895)

Apiaceae: Daucus carota L., Anethum graveolens L.

Apocynaceae: Nerium oleander L.

Asteraceae: $\quad$ Matricaria chamomilla L., Centaurea cyanus L., Achillea millefolium L., Calendula officinalis L., Erigereon annuus (L.) Pers., Helianthus annuus L., Cirsium arvense (L.) Scop., Tagetes patulus L., Serratula tinctoria L., Solidago gigantea Ait., Centaurea jacea L., Leucanthemum ircutianum DC, Artemisia vulgaris L., Dahlia sp., Cinia sp.

Brassicaceae: Brassica napus var. oleifera DC., Cardaria draba (L.) Desv., Sinapis arvensis L., Rorippa sylvestris (L.) Bess, Raphanus landra Moretti, Raphanus raphanistrum L., Capsella bursa-pastoris (L.) Med., Cheiranthus cheiri (L.)

Caryophyllaceae: Melandrium album (Mill.) Garcke, Lychnis flos-cuculi L., Moenchia mantica (L.) Bartl., Sambucus nigra L. Sambucus ebulus L., Dianthus sp.

Chenopodiaceae: Beta vulgaris var. saccharifera Lange

Cichoriaceae: Taraxacum officinale Web., Crepis tectorum L., Crepis jacquini Tausch, Cichorium intybus L.

Convolvulaceae: Convolvulus arvensis L.

Cucurbitaceae: Cucumis melo L., Cucumis sativus L., Cucurbita pepo L.

Cupressaceae: Tuja sp.

Fabaceae: Phaseolus vulgaris L., Trifolium pratense L., Trifolium repens L, Trifolium campestre Schreb., Astragalus glycyphyllos L., Pisum sativum L., Melilotus officinalis (L.) Pall., Lathyrus tuberosus L., Vicia grandiflora Scop., Glycine max (L.) Merr, Mentha sp., Medicago sativa L., Vicia cracca L., Trifolium arvense L.

Geraniaceae: Pelargonium spp.

Hypericaceae: Hypericum perforatum L.

Iridaceae: Gladiolus gandavensis van Houtte

Lamiaceae: $\quad$ Salvia officinalis L., Vicia cracca L., Medicago sativa L., Scutellaria hastifolia L., Stachys annua (L.)

Liliaceae: Allium sp, Lilium sp., Colchicum autumnale $\mathrm{L}$.

Lytraceae: $\quad$ Lytrum salicaria L.

Malvaceae: $\quad$ Malva alcea L.

Onagraceae: Fuschia sp.

Papaveraceae: Papaver rhoeas L. 
Poaceae: Hordeum murinum L., Triticum aestivum L., Zea mays L.

Polyonaceae: Polygonum lapathifolium L.

Plantaginaceae: Plantago altissima L., Plantago major L.

Ranunculaceae: Ranunculus acris L., Ranunculus repens L., Ranunculus arvensis L. Clematis recta $\mathrm{L}$.

Rosaceae: Rosa sp., Potentilla reptans L., Rosacanina L., Potentilla inclinata Vill., Rubus sp., Fragaria sp.

Saxifragaceae: Hydrangea hortensis Sieb.

Scrophulariaceae:Antirrhinum majus L., Linaria vulgaris Mill.

Solanaceae: $\quad$ Solanum tuberosum L., Lycopersicon esculentum Mill., Nicotiana tabacum L., Capsicum annuum L., Petunia hybrida

Violaceae: $\quad$ Viola tricolor L.

3.1.2.9 Frankliniella occidentalis (Pergande, 1895)

Araceae: $\quad$ Cala palustris L.

Apiaceae: $\quad$ Capsicum annuum L.

Asteraceae: Helianthus annuus L.,Chrysanthemum sinense L.

Brassicaceae: Brassica oleracea var. botrytis L.

Caryophyllaceae:Stellaria media (L.) Vill.

Cichoriaceae: Taraxacum officinale Web.

Fabaceae: Galega officinalis L.

Geraniaceae: Pelargonium peltatum L.

Onagraceae: Plobium hirsutum L.

Rosaceae: Rosa sp., Fragaria sp.

Solanaceae: Solanum melongen L, Capsicum annuum L.

3.1.2.10 Frankliniella pallida (Uzel, 1895)

Cichoriaceae: Cichorium intybus L.

Fabaceae: Lathyrus tuberosus L.

Poaceae: Zea mays L.

Rubiaceae: Galium verum $\mathrm{L}$.

Scrophulariaceae: Verbascum nigrum L.

3.1.2.11 Frankliniella tenuicornis (Uzel, 1895)

Asteraceae: Calendula officinalis L., Inula helenium L.

Chenopodiaceae: Beta vulgaris var. saccharifera Lange

Fabaceae: Trifolium repens L., Medicago sativa L., Glycine max (L.) Merr.

Iridaceae: $\quad$ Gladiolus gandavensis van Houtte.

Poaceae: Zea mays L.

Solanaceae: Nicotiana tabacum L., Capsicum annuum L.
3.1.2.12 Kakothrips robustus (Uzel, 1895)

Fabaceae: $\quad$ Lathyrus tuberosus L., Vicia cracca L., Coronilla varia L., Coronilla varia L., Medicago sativa L. Limothrips cerealium Haliday, 1836

Asteraceae: Cirsium arvensis (L.) Scop.

Convolvulaceae: Convolvulus arvensis L.

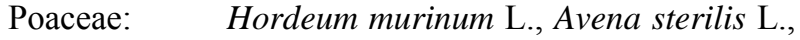
Triticum aestivum L., Avena sativa L.

3.1.2.13 Limothrips denticornis (Haliday, 1836)

Asteraceae: Cirsium arvensis (L.) Scop.

Brassicaceae: Sinapis arvensis L.

Fabaceae: Vicia cracca L.

Poaceae: $\quad$ Hordeum vulgare L., Triticum aestivum L.

3.1.2.14 Odontothrips confusus Priesner, 1926

Fabaceae: Coronilla varia L.

3.1.2.15 Odontothrips loti (Haliday, 1852)

Asteraceae: Achillea millefolium L., Medicago sativa $\mathrm{L}$.

Fabaceae: $\quad$ Coronilla varia L. Medicago sativa L., Dorycnium herbaceum Vill.

3.1.2.16 Oxythrips bicolor (O.M. Reuter,1879)

Asteraceae: Helichrysium italicum (Roth) Mill. corr. Guss., Inula candida (L.) Cass, Inula conyza DC.

Cichoriaceae: Scolymus hispanicus L.

Dipsaceae: Cephalaria leucantha (L.) Schrad.

Lamiaceae: Nepeta cataria L.

Poaceae: $\quad$ Zea mays L.

Rosaceae: Rubus hirtus W. et K.

Scrophulariaceae: Linaria vulgaris Mill.

3.1.2.17 Stenothrips graminum Uzel, 1895

Poaceae: $\quad$ Triticum aestivum L.

3.1.2.18 Sericothrips bicornis (Karny, 1910)

Fabaceae: $\quad$ Trifolium repens L.

3.1.2.19 Thrips flavus Schrank, 1776

Asteraceae: Serratula tinctoria L., Helianthus annus L.

3.1.2.20 Thrips linarius Uzel, 1895.

Asteraceae: Leucanthemum ircutianum DC

Caprifoliaceae: Sambucus nigra L.

Rosaceae: Rosa canina L.

3.1.2.21 Thrips major Uzel, 1895

Asteraceae: Leucanthemum ircutianum DC, Matricaria chamomilla L., Inula helenium L., Centaurea cristata Bartl., 
Centaurea jacea L., Serratula tinctoria

L., Cirsium canum (L.) All.

Boraginaceae: Lithosperum arvense L.

Brassicaceae: Lunaria rediviva $\mathrm{L}$.

Caryophillaceae: Lychnis flos-cuculi L.

Cichoriaceae: Picris hieracioides L., Cichorium intybus L.

Convolvulaceae: Convolvulus arvensis L.

Cucurbitaceae: Cucumis sativus L.

Fabaceae: Trifolium pratense L., Phaseolus vulgaris L.

Liliaceae: Allium scorodoprasum L.

Lythraceae: $\quad$ Lythrum salicaria L.

Malvaceae: $\quad$ Malva sylvestris L.

Poaceae: $\quad$ Triticum aestivum L.

Ranunculaceae: Ranunculus arvensis L., Ranunculus acris $\mathrm{L}$.

Rubiaceae: Galium mollugo L., Galium palustre L.

3.1.2.22 Thrips minutissimus Linnaeus, 1758

$\begin{array}{ll}\text { Asteraceae: } & \begin{array}{l}\text { Leucanthemum ircutianum DC, } \\ \text { Leucanthenum triviale (Gaud.) } \\ \text { Horvatić }\end{array} \\ \text { Iridaceae: } & \begin{array}{l}\text { Gladiolus gandavensis van Houtte } \\ \text { Oleaceae: }\end{array} \\ \text { Olea sativa (Hoffmg. et Lk.) Fiori }\end{array}$

3.1.2.23 Thrips nigropilosus Uzel, 1895

Asteraceae: $\quad$ Acer campestre L.

Cichoriaceae: Sonchus arvensis L., Teraxacum officinale Web.

3.1.2.24 Thrips physapus Linnaeus, 1758

Asteraceae: Leucanthemum ircutianum DC, Calandula officinalis L., Carduus nutans L., Centaurea jacea L. Centaurea scabiosa L., Eupatorium cannabinum L., Helianthus annuus L.

Brassicaceae: Sinapis arvensis L., Sinapis arvensis L.

Cichoriaceae: Crepis biennis L., Tragopogon pratensis $\mathrm{L}$.

Cucurbitaceae: Cucurbita pepo L.

Papaveraceae: Papaver rhoeas L.

Plantaginaceae: Plantago sp.

Ranunculaceae: Ranunculus arvensis L.

Rosaceae: Rubus sp.

Urticaceae: $\quad$ Urtica dioica L.

3.1.2.25 Thrips fuscipennis Haliday, 1836

Asteraceae: Leucanthemum triviale (Gaud.)

Horvatić, Tanacetum vulgare L.

Brassicaceae: Brassica napus var. oleifera DC.

Caprifoliaceae: Sambucus nigra L.

Rosaceae: $\quad$ Rubus idaeus L., Rosa sp.

Saxifragaceae: Hydrangea hortensis Sieb.

Solanaceae: $\quad$ Capsicum annuum L.
3.1.2.26 Thrips tabaci Lindeman, 1888

Apianceae: Daucus carota L., Anethum graveolens L., Eryngium amethystinum L.

Asteraceae: Achillea millefolium L., Leucanthemum ircutianum DC, Calystegia sepium (L.) R.Br. Centaurea cyanus L.,, Matricaria discoidea DC., Cirsium arvense (L.) Scop., Solidago gigantiea Ait., Helianthus annuus L., Erigeron annuus L., Chrysanthemum spp., Solidago irgaurea L. Tripleurospermum indorum (L.) C.H.Schultz., Inula conyza DC., Conyza canadensis (L.) Cronq, Artemisia absinthium L., Inula crithmoides L., Tagetes patulus L.

Brassicaceae: $\quad$ Capsella bursa-pastoris (L.) Med., Sinapis arvensis L.

Boraginaceae: Echium plantagineum L.

Cactaceae: Cactus sp.

Caprifoliaceae: Sambucus nigra L., Sambucus ebulus L.

Chenopodiaceae: Beta vulgaris . var. saccharifera Lange

Cichoriaceae: $\quad$ Crepis setosa Hall. f.

Convolvulaceae: Calystegia sepium (L.) R.Br

Cucurbitaceae: Cucurbita pepo L.

Dipsacaceae: $\quad$ Cephalaria leucantha (L.) Schrad.

Fabaceae: Medicago sativa L., Dorycnium herbaceum Vill., Spartium junceum Glycine max (L.) Merr., Medicago falcata L., Lotus corniculatus L., Vicia sp., Trifolium repens L., Genista tinctoria L., Melilotus alba Med.

Iridaceae: Gladiolus gandavensis van Houtte

Lamiaceae: $\quad$ Lavandula latifolis Med., Origanum vulgare L.

Liliaceae: Allium cepa L., Allium sp.

Malvaceae: Malva sylvestris L.

Moraceae: $\quad$ Ficus carica L.

Oleaceae: Olea sativa (Hoffmg. et Lk.) Fiori

Plantaginaceae: Plantago altissima L.

Poaceae

Triticum aestivum L., Zea mays L., Sorgum bicolor (L.) Moench., Sorgum halepense (L.) Pers.

Polygonaceae: Rumex crispus L.

Punicaceae: $\quad$ Punica granatum L.

Ranunculaceae: Nigella arvensis L.

Resedaceae: $\quad$ Reseda lutea L.

Rosaceae: Filipendula vulgaris Moench, Rubus hirtus W. et K., Fragaria vesca L., Rosa sp.

Rubiaceae: Galium verum L., Galium mollugo L. 
Rutaceae: $\quad$ Ruta graveolens L.

Saxifragaceae: Hydrangea hortensis Sieb.

Scropholariaceae: Verbascum sinuatum L., Verbascum sinuatum L.

Solanaceae: $\quad$ Lycopersicon esculentum Mill.

3.1.2.27 Taeniothrips atratus (Haliday, 1836)

Caryophllyaceae: Melandrium album (Mill.) Garcke.

Geraniaceae: Pelargonium spp.

Lamiaceae: Lavandula latifolia Med., Stachys annua (L.)

Poaceae: Dactylis glomerata L

Solanaceae: $\quad$ Solanum tuberosum L.

3.1.2.28 Taeniothrips vulgatissimus (Haliday, 1836)

Corylaceae: $\quad$ Corylus avellana L.

3.1.2.29 Taeniothrips inconsequens (Uzel, 1895)

Asteraceae:

Artemisia absinthium L., Centaurea

diffusa Lam.

Cichoriaceae: $\quad$ Picris hieracioides L.

Lamiaceae: Stachys palustris L.

Poaceae: $\quad$ Lolium perenne L.

Ranunculaceae: Clematis flammula L.

3.1.2.30 Thripidae larvae

Aceraceae:

Apiaceae:

Asteraceae: Leucanthemum triviale (Gaud.)

\section{Acer campestre L.}

Daucus carota L. Horvatić, Matricaria chamomilla L., Inula britannica L., Inula helenium L., Arctium lappa L.

Boraginaceae: Echium vulgare L.

Brassicaceae: Raphanus landra Moretti, Raphanus raphanistrum L., Rorippa sylvestris (L.) Bess.

Caryophyllaceae: Lychnis flos-cuculi L.

Chenopodiaceae: Beta vulgaris var. saccharifera Lange

Cichoriaceae: Taraxacum officinale Web.

Convolvulaceae: Convolvulus arvensis L.

Fabaceae:

Medicago sativa L. Melilotus officinalis (L.) Pall., Vicia sp., Vicia grandiflora Scop., Medicago sativa L., Glicyne max (L.) Merr. Lathyrus tuberosus L., Trifolium pratense L., Medicago falcata L., Lotus corniculatus L., Trifolium pratense $\mathrm{L}$.

Malavaceae: $\quad$ Malva sylvestris L.

Oleaceae: $\quad$ Ligustrum vulgare L.

Piniaceae: Pinus sp.

Poaceae: $\quad$ Triticum aestivum L., Avena sativa L., Hordeum vulgare L., Zea mays

Ranunculaceae: Ranunculus acris L.

L., Sorgum bicolor (L.) Moench.

Rosaceae: Rubus sp.
Rubiaceae: $\quad$ Galium mollugo L.

\subsection{Suborder Tubulifera}

3.2.1 Family Phlaeothripidae

3.2.1.1 Bolothrips icarus (Uzel, 1895)

Caryophyllaceae: Melandrium album (Mill.) Garcke

Lamiaceae: Mentha pulegium L.

Malvaceae: Malva sylvestris L.

Plantaginaceae: Plantago altissima L.

Poaceae: $\quad$ Festuca heterophylla Lam.

Ranunculaceae: Clematis flammula L.

3.2.1.2 Cryptothrips nigripes (O. M. Reuter, 188O)

Avagaceae: Jucca sp.

3.2.1.3 Cephalothrips monilicornis O.M. Reuter 1885

Asteraceae: Helianthus annuus L.

Brassicaeae: Lunaria rediviva $\mathrm{L}$.

Chenopodiaceae: Beta vulgaris var. saccharifera Lange.

Cucurbitaceae: Cucurbita pepo L. Picris sp.

Dipsacaceae: Scabiosa sp.

Fabaceae: $\quad$ Vicia cracca L., Phaseolus vulgaris L., Glycine max (L.) Merr.

Poaceae: Zea mays L. Triticum aestivum L., Lolium perenne L., Trifolium pratense L., Avena sativa L., Sorgum halepense (L.) Pers.

Ranunculaceae: Ranunculus arvensis L.

Scrophulariaceae: Linaria vulgaris Mill.

3.2.1.4 Haplothrips aculeatus (Fabricius, 1803)

Apiaceae: Daucus carota L.

Asteraceae: Cirsium arvensis (L.) Scop,

Cichoriaceae: $\quad$ Erigeron annuus L

Fabaceae: $\quad$ Phaseolus vulgaris L., Medicago sativa $\mathrm{L}$.

Poaceae: $\quad$ Zea mays L., Triticum aestivum L., Avena sativa L., Dactylis glomerata L.

3.2.1.5 Haplothrips leucanthemi (Schrank, 1781)

Asteraceae: Leucanthemum ircutianum DC, Matricaria chamomilla L.

Fabaceae: $\quad$ Trifolium pratense L., Glycine max (L.) Merr.

3.2.1.6 Haplothrips minutus (Uzel, 1895)

Asteraceae: $\quad$ Erigeron annuus L., Tagetes patulus L., Solidago gigantea Ait.

Caryophyllaceae: Lychnis flos-cuculi L.

Cichoriaceae: Cichorium intybus L.

Cucurbitaceae: Cucumis sativus L.

Fabaceae: Lathyrus tuberosus L., Medicago sativa L., Trifolium repens L, 


\begin{tabular}{|c|c|}
\hline & $\begin{array}{l}\text { Trifolium pratense L. Glycine max } \\
\text { (L.) Merr. }\end{array}$ \\
\hline Liliaceae: & Lilium sp., Allium sp. \\
\hline Oleaceae: & Olea sativa (Hoffmg. et Lk.) Fiori \\
\hline Pinaceae: & Pinus sp. \\
\hline Poaceae: & $\begin{array}{l}\text { Zea mays L., Avena sativa L., Lolium } \\
\text { perenne L., Hordeum vulgare L., } \\
\text { Triticum aestivum L. }\end{array}$ \\
\hline Polygonaceae: & Rumex sanguineus L. \\
\hline
\end{tabular}

3.2.1.7 Haplothrips niger (Osborn, 1883)

Asteraceae: $\quad$ Matricaria chamomilla L. Caryophyllaceae: Melandrium album (Mill.) Garcke

Fabaceae: Vicia cracca L.

Plantaginaceae: Plantago altissima L.

3.2.1.8 Haplothrips setiger Priesner, 1921

Asteraceae: Matricaria chamomilla L.

Poaceae: $\quad$ Triticum aestivum L.

3.2.1.9 Haplothrips tritici Kudjumov, 1912

Asteraceae: $\quad$ Matricaria chamomilla L.

3.2.1.10 Liothrips vaneeckei Priesner, 1920

Asteraceae: Helianthus annuus L.,Chrysanthemum spp., Achillea millefolium L.

Cichoriaceae: Sonchum arvensis L.

Fabaceae: Trifolium pratense L., Trifolium repens L., Lathyrus tuberosus L., Glycine max (L.) Merr.

Fagaceae: Quercus pubescens Willd.,

Liaceae: Colchicum autumnale L.

Onagraceae: Epilobium hirsutum L.

Plantaginaceae: Plantago sp., Plantago lanceolata L.

Poaceae: Zea mays L. Hordeum murinum L.,. Triticum aestivum L., Avena sativa L., Lolium perenne L., Sorgum halepense (L.) Pers.

Rosaceae: Rubus sp.

Solanaceae: Nicotiana tabacum L.

3.2.1.11 Neoheergeria verbasci (Osborn, 1886)

Asteracea: $\quad$ Centaurea jacea L.
Brassicaceae: Capsella bursa-pastoris (L.) Med.

Polygonaceae: Rumex obtusifolius L.

Poaceae: Avena sativa L.

3.2.1.12 Phlaeothrips coriaceus Haliday, 1836

Poaceae: $\quad$ Triticum aestivum L.

\subsubsection{Poecilothrips albopictus Uzel, 1895}

Rosaceae: Malus sp.

3.2.1.14 Xylaplothrips fuliginosus Schille, 1910

Apiaceae: Daucus carota. L.

Asteraceae: Leucanthemum ircutianum DC, Matricaria chamomilla L., Achillea millefolium L., Centaurea cristata Bartl., Centaurea jacea L., Inula crithmoides L., Helichrysium italicum (Roth) Mill. corr. Guss, Tanacetum vulgare L.

Brassicaceae: $\quad$ Sinapis arvensis L.

Caprifilaceae: Sambucus ebulus L.

Cichoriaceae: Crepis biennis L.

Corylacea: $\quad$ Carpinus betulus L.

Dipsacaceae: Cephalaria leucantha (L.) Schrad.

Plantaginaceae: Plantago lanceolata L.

Solanaceae: Nicotiana tabacum L.

3.2.1.15 Phlaeothripidae larvae

Apiaceae: Daucus carota L.

Asteraceae: Triticum aestivum L., Matricaria chamomilla L., Centaurea cyanus L., Achilea millefolium L., Inula candida (L.) Cass.

Dipsacaceae: Scabiosa sp.

Fabaceae: $\quad$ Trifolium repens L.

Malvaceae: Malva sylvestris L.

Plantaginaceae: Plantago lanceolata L.

Pintaceae: Pinus sp.

Poaceae: $\quad$ Triticum aestivum L, Agropyron pungens (Pers.) Roem. et Schult, Zea mays L. Avena sativa L, Hordeum vulgare L.

\section{DISCUSSION AND CONCLUSIONS}

Thrips species determined in the investigation belong to the three families: Aeolothripidae, Thripidae, and Phlaeothripidae. The presence of thrips from those three families was confirmed by Mound et al. (1976.) in most European countries. The host plants of thrips determined in the investigations belongs to 49 botanical families.

Thrips species from Aeolothripidae family were presented on $32 \%$ of botanical families within our research, mostly on families Fabaceae, Asteraceae and
Brassicaceae. Aeolothrips intermedius - important predator of thrips and mites (Trdan et al., 2005), which was found on host plants from 15 different botanical families, was the most abundant species from Aeolothripidae family.

Thrips specimens from Thripidae family were the most abundant; they were from 14 genera and 30 species. They were presented on $89 \%$ of botanical families within our research. Genus Thrips with 10 species, and the most abundant species T. tabaci, were determined

Acta agriculturae Slovenica, 93 - 3, september 2009 
on 30 botanical families with the highest number on Asteraceae and Fabaceae. The second most abundant thrips species was Frankliniella intonsa. This species was presented on $32 \%$ of examined samples, on 29 botanical families, mainly on Asteraceae and Fabaceae, followed by Brassicaceae, Caryophyllaceae, Rosaceae, Lamiaceae, and Ranunculaceae. Both Thysanoptera species are also known as widely distributed insects in Slovenia (Trdan, 2003), Serbia (Andjus and Trdan, 2005ab), Hungary (Jenser and Czencz, 1988) and in many other European countries with continental climate, while only Thrips tabaci is known as important pest of some vegetable plants (Trdan et al., 2007).

Family Phlaeothripidae was presented with 9 genera and 14 species. Their representatives, which do not belong among important pest species in Croatia, their neighbouring countries and also some Mediterranean countries (Garcia-Fayos and Goldarazena, 2008), were presented on 27 plant families, mainly on Poaceae (40 $\%)$, Asteraceae (33\%), and Fabaceae (26\%). The most abundant species from Phlaeothripidae family, which representatives are potential pollinator of their host plants, was Haplothrips minutus, which was found in host plants from 10 botanical families.

On the basis of results of present study we can conclude that the majority of Thysanoptera species are polyphagous. This characteristic allow them survival in different agroecosystems, where some of these insects perform the permanent threat to cultivated plants. Therefore the knowledge on host plants of thrips species is highly advantageous in research work as well as in implementation of sustainable methods of thrips control (e.g. trap crops [Buitenhuis et al., 2007], intercropps [Trdan et al., 2006] etc.) in food production systems.

\section{ACKNOWLEDGEMENT}

The authors thank to Prof. Ana Skender for determination of host plants of thrips.

\section{REFERENCES}

Andjus Lj. (1997): Check-list of Thysanoptera in the former Yugoslavia. Acta Entomol. Serb., 2: 117-136.

Andjus, Lj., Trdan, S. (2005a): Duvanov trips (Thrips tabaci Lindeman), najštetnina vrsta tripsa na otvorenom prostoru. Biljn. lek., 33: 395-400.

Andjus, Lj., Trdan, S. (2005b): Tripsi na lucerki. Biljn. lek., 33: 538-542.

Buitenhuis, R., Shipp, J. L., Jandricic, S., Murphy, G., Short, M._(2007): Effectiveness of insecticide-treated and nontreated trap plants for the management of Frankliniella occidentalis (Thysanoptera: Thripidae) in greenhouse ornamentals._Pest Manag. Sci., 63: 910-917.

Ciglar, I., Medin, A., Bušić, Z., Brekalo, J., Ljubićić, M. (1984): Taeniothrips meriodinalis (Priesner.) novi štetnik bresaka u nas. Glas. zašt. bilja, 12: 423-424.

Ciglar, I., Žužić, I. (1990): Lozin trips - Drepanothrips reuteri Uzel sve ćešći štetnik u vinogradima. Glas. zaštite bilja, 12: $422-423$.

Domac, R. (1994): Flora Hrvatske, priručnik za određivanje bilja. Školska knjiga, Zagreb: 1-504.

Ehrendorfer, F. (1973): List der Gefäßpflanzen Mitteleuropas. Gustav Fischer Verlag, Stuttgart: 1-318.

Garcia-Fayos, P., Goldarazena, A. (2008): The role of thrips in pollination of Arctostaphyllos uva-ursi. Int. J. Plant Sci., 169: 776-781.

Janežič, F. (1991): Prispevek k poznanju tripsov ali resarjev (Thysanoptera) na rastlinah v Sloveniji. Zb. Bioteh. Fak. Univ. Ljubl., 57: 169-178.
Janežič, F., Maček, J. (1993): Proširenost kalifornijskog tripsa (Frankliniella occidentalis /Perg/) u Sloveniji. Glas. zašt. bilja, 11-12: 310-311.

Jenser, G. (1982): Tripszek - Thysanoptera. Magyarosazág Állatvilága Fauan Hungariae, Akadémiai Kiadó, Budapest: 1-192.

Jenser, G. (1986): Data to the Thysanoptera fauna of the Kiskunsà national park. The Fauna of the Kiskunsàg National Park: 107-111.

Jenser, G., Czencz, K. (1988): Thysanoptera species occurring frequently on cultivated plants in Hungary. Acta Phytopathol. Entomol. Hung., 23: 285-289.

Jenser, G. (1990): Host preference of some Thysanoptera species living in Fabaceae flowers. In: Proceed. $3^{\text {rd }}$ Int. Symp. Thysanoptera, Kazimircz Dolny, Poland: 83-89.

Jenser, G., Balogh, E. (1992): Predatory Thysanoptera in Hungarian Orchards. Növvéd., XXVIII, 9: 352-255.

Jenser, G.. Tusnádi, C. K. (1989): A Nyugati virágtripz (Frankliniella occidentalis Pergande) megjelenése Magyarorzágon. Növvéd., XXV, 9: 389-393.

Jenser, G., Vierbergen, B., Szénási, Á. (2007): Thysanoptera larvae living on chickweed (Stellaria media Linnaeus) under continental climatic conditions. Acta Phytopathol. Entomol. Hung., 42: 387-390.

Kovačević, Ž. (1964): Kretanje, brojnost i zastupljenost nekih rodova insekata na lucerištima, djetelištima i livadama. Zašt. bilja 82, XV: 667-685. 
Masten, R. (1983): Resičari štetnici plodova breskve. Glas. Zašt. Bilja, 10: 395-396.

Morison, G., Tanasijević, N. (1966): The frequency of Thysanoptera on some crop plants of Yugoslavia. The Entomologist 99: 28-43.

Moritz, G. (1994): Pictorial key to the economically important species of Thysanoptera in Central Europe. Bull. OEPP/EPPO Bull. 24: 181-208.

Mound, A. L., Morison D. G., Pitkin, R.B., Palmer, M. J. (1976): Thysanoptera. Handbook for the Identification of British Insects. Royal Entomological Society of London 1, 11: 8-18.

Mound A. L. (1997): Thrips as crop pests. Biological Diversity. CAB Int.: 198-215.

Okumura, T. G., Papp, S. C. (1991): Insect and Mite Pests in Food. Thrips (Thysanoptera): 403-414.

Raspudić, E. (1996): Fauna tripsa (Insecta: Thysanoptera) u Republici Hrvatskoj. Dr. diss, Fac. Agric. Osijek: 1-139.

Raspudić, E., Ivezić, M. (1999): Biljke domaćini i nalazišta resičara Thrips tabaci Lindeman 1888 (Thysanoptera, Thripidae) u Hrvatskoj. Entomologia Croatica 4, 1-2, 5762 .

Raspudić, E., Ivezić, M., Jenser, G. (2003): Check list on Thysanoptera in Croatia. Entomol. Croat., 7: 35-41.

Šimala, M. (1991): Frankliniella occidentalis (Perg.) (Thysanoptera, Thripidae) novi štetnik povrća i ukrasnog bilja u Jugoslaviji. Agron. Glas., 4-5: 255-258.

Trdan S. (2002): Vrednotenje morfološke in genetske raznolikosti populacij gospodarsko pomembnih vrst resarjev (Thysanoptera) v Sloveniji. Dokt. dis., Ljubljana, Univ. Ljubl., Bioteh. fak.: 1-90.
Trdan, S. (2003): The occurrence of thrips species from the Terebrantia suborder on cultivated plants in Slovenia. Res. Rep., Biotech. Fac., Univ. Ljubl., Agric. issue 1, 81: 57-64.

Trdan, S., Andjus, Lj., zur Strassen, R. (2003): Chronology of thripsological activities and comparison of check-lists on Thysanoptera in Slovenia and FR Yugoslavia. Acta Entomol. Slov., 11: 61-70.

Trdan, S., Andjus, Lj., Raspudić, E., Kač, M. (2005): Distribution of Aeolothrips intermedius Bagnall (Thysanoptera: Aeolothripidae) and its potential prey Thysanoptera species on different cultivated host plants. J. Pest Sci., 78: 217-226.

Trdan, S., Žnidarčič, D., Valič, N., Rozman, L., Vidrih, M. (2006): Intercropping against onion thrips, Thrips tabaci Lindeman (Thysanoptera: Thripidae) in onion production: on the suitability of orchard grass, lacy phacelia, and buckwheat as alternatives for white clover. J. Plant Dis. Prot. 113: 24-30.

Trdan, S., Valič, N., Žnidarčič, D. (2007): Field efficacy of deltamethrin in reducing damage caused by Thrips tabaci Lindeman on early white cabbage. J. Pest Sci., 80: 217 223.

zur Strassen, R. (1981): Erste daten zur Thysanopteren-fauna des nordwestlichen Istrien (Jugoslawien). Acta entomol. Jugosl., 17: 143-151.

zur Strassen, R. (1984): Zur Thysanopteren-Faunistik des Alpen-Vorlandes von Slowenien, Nebst Einer Check-List der Fransenflügler-Arten von Jugoslawien. Acta entomol. Jugosl. 20: 31-51. 